\title{
EuroPCR 2021: the next milestone on the road to excellence in postgraduate education
}

\author{
Jean Fajadet, Bernard Prendergast, William Wijns \\ On behalf of the PCR Courses Directorship
}

\section{Welcome to the EuroPCR 2021 issue of Eurolntervention}

What is the future of postgraduate education in the field of interventional care in the COVID era? The "what" and the "why" are the same, but the "how" is changing at an accelerated pace, pushed on by the strong tailwind of digital transformation. While highlighting some of the unique and novel features of the 2021 EuroPCR vintage, we are taking the opportunity in this editorial to share our vision of the future PCR educational experience.

Like nearly everybody else, the pandemic took us by surprise in 2019. But thanks to agile and adaptable support from the Europa Group - the backbone organisation of PCR - we were able to stay connected with the community, largely through PCRonline, the EuroIntervention journal and social networks.

We have learnt a lot during the organisation of initiatives such as "Facing COVID with PCR", digital replacement events across the portfolio of PCR Courses, and a large series of topical webinars. Following the cancellation of physical meetings, digital activity in 2020 actually increased very significantly compared to 2019. For example, an $18 \%$ rise in the number of page views for EuroIntervention and 54\% more followers on Twitter, 30\% more traffic for PCR Webinars, and a record number of 15,025 participants in the PCR e-Course (with an average total connection time exceeding 6 hours). Compared to 2019, we have also seen a large increase in the online participation of healthcare professionals from Argentina, Brazil, India, Indonesia, Mexico, and Saudi Arabia colleagues who do not usually attend the on-site meeting in Paris as well as the United States and the United Kingdom.

These impressive numbers highlight the appetite for exchange, communication and opportunities for greater outreach, but did not spare us from performing a critical qualitative evaluation.

\section{Qualitative evaluation of the 2020 experience and needs assessment}

Key learnings from PCR's initial experience with digital media (Table 1) confirm the strong links connecting our community, PCR Companions, nurses, physicians, allied professionals and MedTech industry partners.

The community far prefers live interaction to recorded events; the direct transfer of content prepared for physical meetings onto the web does not work well. A totally new skill set is required, not only in terms of presentation but also in the adaptation of visuals for projection on small computer screens or mobile phones. Even though the fundamentals of adult learning remain the same, the shift to digital teaching demands a cultural change in "how" education is delivered. 
Table 1. Key learnings from PCR educational activities in 2020. Achievements from 2020: what did we learn?

Relevant content attracts a committed PCR community

Quality of users' experience on digital format counts

Easy navigation

Renewed support from industry partners

Permanent exchanges

Agility and adaptability
Strong desire for more interaction

Live preferred over recorded sessions

Participants' experience: listening vs sharing

Digital is not forgiving

Sharper/more specific content needed

Adjusted visuals needed

TV studio skills to be acquired

Fewer opportunities to expose participants as presenters

Digital education: the fundamentals are exactly the same....but everything else is totally different

Table 2. Multiple-source needs assessment on behalf of all stakeholders.

PCR Board \& Faculty members' needs

2 Sounding Boards with $60+$ physicians

To empower more communities (NextGen, National Societies...)

To keep working together on reinventing education, innovating considering the

worldwide changes and context

To deliver the EuroPCR 2021 programme whatever the situation in May 2021 (avoiding any risk of late cancellation)

\section{PCR community needs}

Satisfaction surveys post e-Course, webinars and via PCRonline

To benefit from more opportunities to present their work (cases and abstracts)

To live the PCR learning experience again

To have access to "on demand" resources and live interactions

To meet with their peers

To overcome "digital fatigue"
Through needs assessment (Table 2), we have also learned that relevant content fails to catch attention when the teaching is not practical, interactive, impactful or patient-centred. Further obvious limitations of current digital events include the lack of "face-to-face" interaction, non-verbal communication and opportunities for networking.

The community is sending a strong message that reinforces our commitment to PCR's mission for the past 30 years - continued search for excellence in education. The "what" and the "why" should remain the same, while taking advantage of the opportunities provided by digital transformation to offer an enduring and self-directed educational experience to a much larger audience than previously envisaged.

As a consequence, we have instituted a re-invention and redesign process focused on the "how" that aims to combine the best of digital (the present) and in-person (the past and future) meetings. Specifically, EuroPCR 2021 is designed to address 3 major goals:

- Bring the community of practitioners "back on stage"

- Take advantage of digital technology to expand outreach and create a continuum of education

- Advance the quality of learning to the next level through innovative broadcast programmes

\section{EuroPCR 2021 and future PCR Courses}

The Course on 18-19-20 May will be digital and delivered via an intuitive platform to create an inclusive congress environment with "in-person" windows (Hubs and Pods) located worldwide (Figure 1).

Depending on prevailing pandemic conditions, participants will have the choice of participating in the Course alone via virtual connections, or of joining their colleagues in nearby Hubs and Pods. A continuum of education will be achieved by upstream release of content, including clinical cases, PCR Clinical Research modules, the "PCR's Got Talent" programme, the EAPCI/PCR Fellows Course or the course PCR Peripheral Interventions for Beginners. The entire EuroPCR 2021 content will remain available on demand for 3 months following the event.

Without going into the details of the technically complex links between studios, Hubs and Pods, the output will be transparent for the participant, with dynamic teaching, diverse exchanges, and ample opportunity for live interaction via chat or in person. PCR Hubs located worldwide will allow us to share the voice of local communities on a truly global scale.

What is essential to realise, however, is the cultural change. We are moving away from the static concept of successive sessions, with Chairpersons, PowerPoint lectures and Panellists. Instead, the 5 studios will broadcast relevant content in a TV programme format connected to a Local Hub, building on successful experimentation of this concept during the 2020 PCR Valves e-Course (Figure 2).

We are very grateful to all our colleagues around the globe who have agreed to engage in this cultural change and remain committed to learning and adopting entirely new skills, whilst advancing their training in VITAL facilitation (VIsionary Transformative Adult Learning) to the next level.

Together with all PCR teams and the Europa Group, we look forward to your involvement in this new educational experience. Make sure to mark your diary and prepare your active contribution to this unique event. 


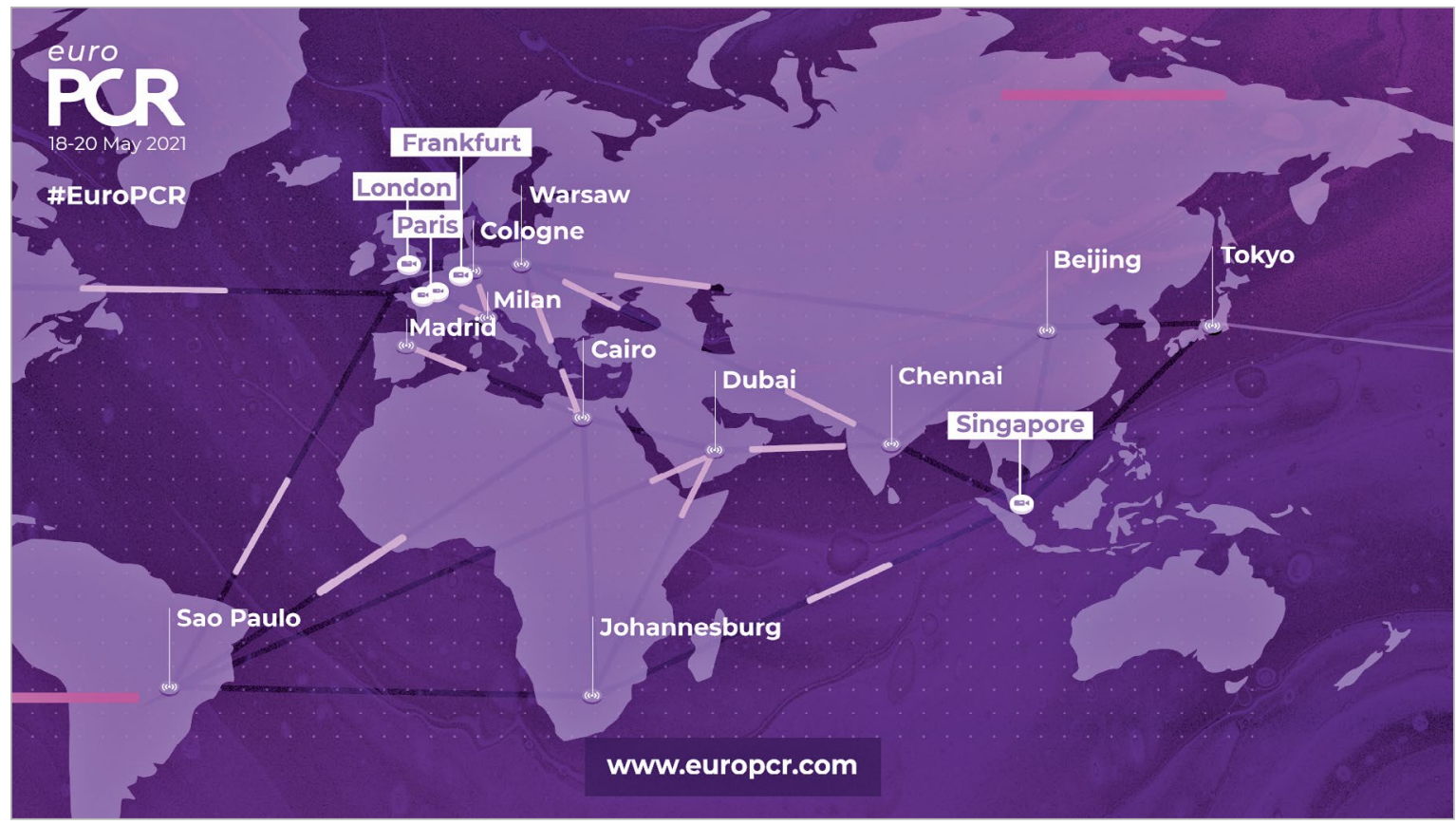

Figure 1. A global interactive network unifying local physical meetings.

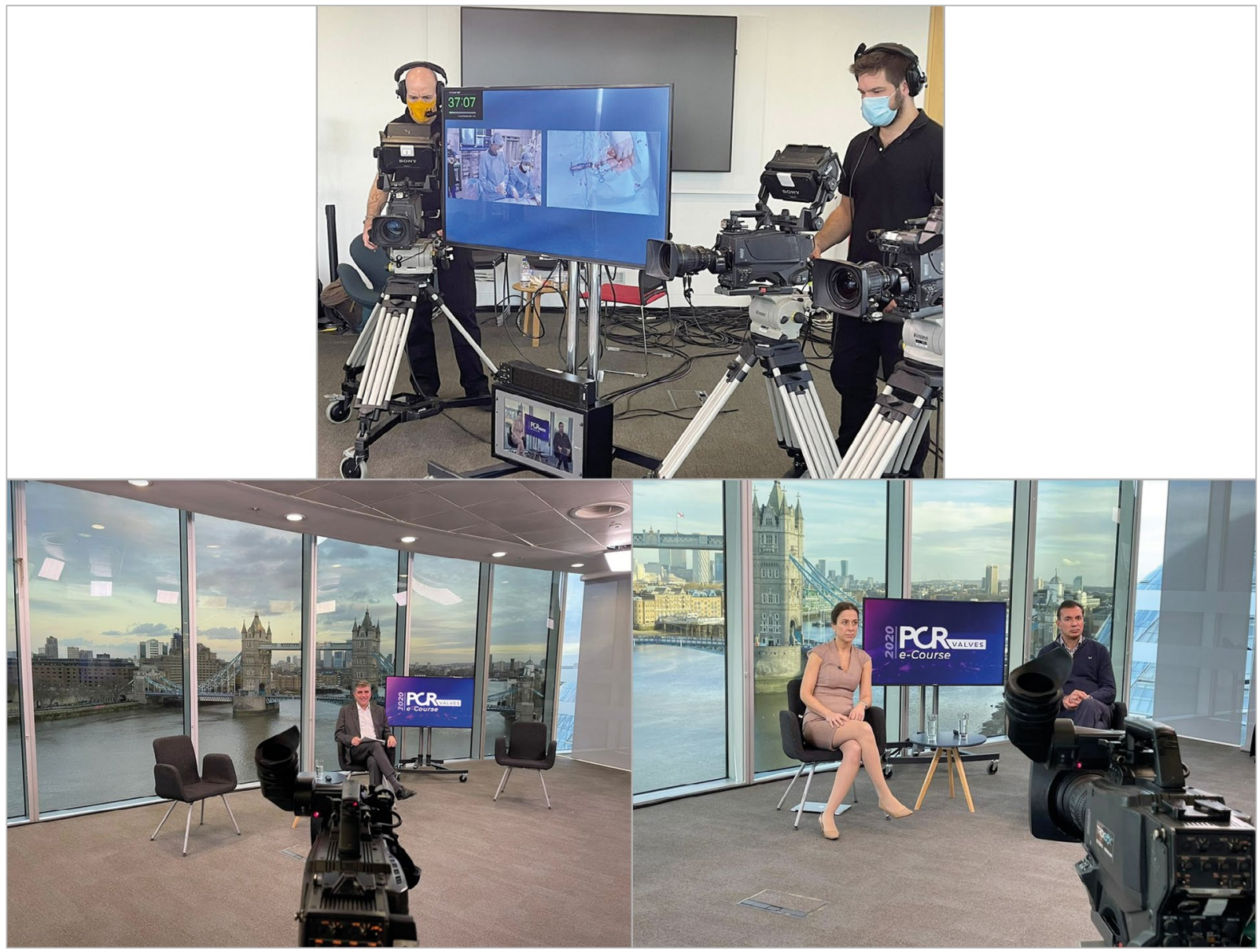

Figure 2. "Behind the camera" view of the London Bridge studio during the November 2020 PCR Valves e-Course. From a standard session to a 3-hour TV programme: TV studios offer live broadcasts and alternating media (pre-recorded interviews, clips and jingles, international coverage, virtual LIVE cases, and more). Interaction is key with more time for discussion and non-stop chat in parallel during the programme. 